\title{
Stellar wind and supernova feedback from massive stars
}

\author{
Julian M. Pittard and Hazel Rogers \\ School of Physics and Astronomy, The University of Leeds, Leeds, LS2 9JT, UK \\ email: jmp@ast.leeds.ac.uk
}

\begin{abstract}
We have constructed three-dimensional hydrodynamical models to simulate the impact of massive star feedback, via winds and SNe, on inhomogeneous molecular material left over from the formation of a massive stellar cluster. We are studying the timescales for the molecular material to be removed from the environment of a massive stellar cluster and the mass and energy fluxes into the wider environment.
\end{abstract}

Keywords. hydrodynamics, ISM: bubbles, HII regions, supernova remnants

\section{Introduction}

Massive stars blow powerful winds and explode as supernovae. However, the degree to which these inputs couple to the clumpy and inhomogenous molecular clouds which initially surround a cluster of massive stars is currently ill-determined. We are using three-dimensional hydrodynamical simulations to elucidate this.

\section{Model and results}

In our model 3 O-stars are located at the centre of a giant molecular cloud (GMC) clump of radius $4 \mathrm{pc}$ and mass $3000 \mathrm{M}_{\odot}$. The stars collectively drive a cluster wind, and transition through 3 evolutionary phases (main sequence - red supergiant - Wolf-Rayet) before exploding.

We find that hot, high speed gas flows away from the cluster in low-density channels opened up by the stellar winds. Mass is loaded into these flows from the ablation of dense clouds embedded within them and from material stripped from the dense gas which confines and directs the flows. Dense parts of the clump can shield and protect less dense material in their "shadow". The rate of ablation appears to be roughly constant during the first few Myr of the cluster evolution, and the mass flux into the wider environment can be 3 orders of magnitude greater than the combined mass-loss rates of the stars. About one third of the input wind mechanical energy is radiated away. In contrast, almost all of the energy from each SN explosion escapes into the wider medium. This is due to the high porosity of the dense molecular material at this stage which allows the SN blast to rip through the cluster in a largely unimpeded fashion. A detailed analysis of our results is currently underway, and in future work we plan to study a range of clump and cluster parameters. 